\title{
Los Sistemas Dinámicos como Teoría Cognitiva
}

\section{Dynamic Systems as Cognitive Theory}

\author{
Marcelo Arce Fonollá \\ Universidad de Chile
}

(Recepción: Noviembre 2005 - Aceptación: Diciembre 2005)

\begin{abstract}
El conocimiento científico se basa en los sentidos, pero la ciencia explica los fenómenos naturales por medio de procesos reversibles donde el tiempo humano no tiene cabida. Los sistemas dinámicos, por su naturaleza multidimensional, dan cuenta de la irreversibilidad y la evolución, lo que los acerca a las ciencias humanas y da origen a un modelo de cognición más cercano a las causas físicas que los modelos anteriores computacional y conexionista.
\end{abstract}

Palabras clave: sistemas dinámicos, multidimensional, cognición.

\begin{abstract}
Scientific knowledge is based on the senses; however, science explains natural phenomena through reversible processes that leave no room for the human concept of time. Dynamic systems, with their multidimensionality, express irreversibility and evolution, which bring them closer to human sciences and give rise to a cognitive model that is nearer physical causation than the previous computational and connectionist models.
\end{abstract}

Key words: Dynamic systems, multidimensional, cognition

\section{Antecedentes}

Teorías de sistemas dinámicos se han elaborado desde muy antiguo. Ya Lucrecio, discípulo de Epicuro, anticipó una "teoría del todo" que se desarrollaba sobre la base de sus postulados acerca de la existencia de átomos y vacío. Representando la idea de que todo es infinito, y en un esfuerzo por armonizar el dinamismo atómico, postulaba que los acoplamientos de átomos explicaban tanto la vida como las libertades humanas, y le permitían plantear además la idea de una selección natural. Idea esta última que, como ya descubrió Bergson, anticipaba a Darwin en la definición del "concepto" de evolución.

El poeta Paul Valery contribuyó también a la teoría de los sistemas dinámicos, como resalta el matemático Rene Thom, al descubrir la audaz trama entre ciencia y arte, en un legado que sólo sería comparable a los de Leonardo, Goethe o Pascal: "Finalmente, la idea de una teoría de dinámica universal, capaz de aplicarse indiferentemente a los procesos mentales, la morfogénesis biológica, y a ciertos fenómenos de conflicto de la naturaleza inanimada como los cambios de fase, parece sustentar bien el proyecto valeriano, a semejanza del principio

1 Licenciado en Arte, Universidad de Chile. Magíster (c) en Estudios Cognitivos, Universidad de Chile. E-mail: marceloarce@netscape.net de la teoría de las catástrofes" (Schwartzman, 2000). En efecto, para Valery el cerebro sería un lugar de cambios incesantes, "habitado por la inestabilidad misma" (Schwartzman, 2000), regido por una mecánica mental donde la diferencia orden-desorden es equivalente a la diferencia de temperatura en una máquina.

\section{El problema del espacio-tiempo}

Sin embargo, es en el siglo $\mathrm{XX}$ donde esas teorías decantan y se introducen en los procesos cognitivos. Parece existir, como diría Einstein, un "insatisfactorio estado intermedio" de nuestra relación con la realidad. Sin embargo, respecto a la concepción del tiempo y el espacio, podría ocurrir que una multiplicidad de esquemas conceptuales (y no sólo uno) fuera esencial a toda construcción mental adecuada a los fenómenos. El misterio de las cualidades sensoriales radica en que, como Schrödinger escribe: "todo conocimiento científico se basa en los sentidos, y no obstante, las descripciones científicas de los procesos naturales así elaboradas carecen de todas las cualidades sensoriales, por lo que no pueden dar cuenta de ellas" (Jaques, 1984), la vivencia primaria del espacio y el tiempo es la de un continuo espacio-tiempo indiferenciado. Vivimos en un mundo mentalmente construido de acción y de cambio, y no tropezamos con grandes dificultades hasta 
que intentamos enunciarlo, ya sea poética o científicamente.

La jerarquización de los eventos complejos corresponde a una estructura mental, en la que asumimos la existencia de un agente organizador centralizado. Sin embargo, existen instancias, como el tiempo, que si bien organiza la existencia en un "antes" y un "después", no la articula en torno a la percepción de la modificación espacial, sino que "es" el cambio. Tal vez por esto la teoría cuántica resulte aún tan obscura para la mayoría de la gente, ya que si bien en términos operativos aún se emparenta con la teoría de Newton (la función de onda (j) es muy similar a la trayectoria en la mecánica clásica), no se refiere a una linealidad observable, la onda no es visible como sí lo es un móvil que se desplaza.

John Von Neumann hizo la presentación de la teoría cuántica cinco años después de ser elaborada por Schrödinger, en 1932, instalando una visión dualista: "por una parte, tenemos la ecuación de Schödinger que rige la función de onda, y que (al igual que la ecuación de Newton) es determinista y simétrica en relación al tiempo. Por otra parte tenemos la reducción de la función de onda, que transforma un caso puro en una mezcla y que, asociada al proceso de medición, es irreversible" (Prigogine, 1996). Su aplicación más importante es en la teoría de la relatividad general, la cual para poder sustentarse hubo de cambiar el espacio euclidiano por el riemiano (o curvo).

Se puede resumir la teoría general de la relatividad como aquella ley que confirma la posibilidad del avance continuo del tiempo en un continuo espacio tridimensional curvado por la presencia de materia. "Pero curvando el espacio más y más se llega inevitablemente a un punto singular donde no hay definición ni para tiempo ni para espacio, que en esas condiciones críticas dejan de ser continuos" (PenroseHawking, 1996).

Queda la ruina de la trama espacio-temporal destruida por la gravitación y la presencia de los agujeros negros, singularidades paradójicamente antagónicas a toda continuidad (aun ante la reciente retractación inédita de Hawking). Las singularidades espacio-temporales como estaban definidas hasta ahora correspondían a la divergencia de la curvatura del espacio y estaban presentes en el Big Bang, los Hoyos Negros y el Big Crunch.

\section{Resonancias, niveles y emergencia}

Partiendo de una pregunta acerca de la estabilidad del sistema solar, Henri Poincaré abrió el camino para tratar los problemas de estabilidad en sistemas dinámicos complejos. En un universo estable, donde pequeños cambios en las condiciones iniciales sólo traen pequeñas alteraciones en el comportamiento del sistema considerado y donde, por tanto, pueda existir "el demonio de Laplace" que es capaz de predecir el futuro siempre que conozca el estado presente del universo con el suficiente grado de precisión el problema simplemente no se presenta.

Pero en un universo inestable, tal tipo de predicción determinista es imposible; y el universo en el cual vivimos realmente es un universo inestable, irreversible y, por tanto, evolutivo (asociado a la flecha de tiempo). Es de sentido común que el pasado no puede disolverse como si no hubiera existido, sino que determina irreversiblemente el presente que estamos viviendo; sin embargo, en las ciencias es común enfrentarse a la reversibilidad de los procesos, como si fueran lineales.

Poincaré estableció una diferencia entre sistemas estables e inestables (o sistema no integrable): si un sistema se puede reducir, eliminando la energía potencial, de modo que la resultante sea equivalente a un sistema en el que las partículas no tienen interacción entre ellas, dicho sistema es integrable, sin embargo en él tampoco hay vida o auto organización. Por el contrario, la mayoría de los sistemas es no integrable y esto se debe a las "resonancias en los grados de libertad del sistema". Del mismo modo que, en la música, se suman las vibraciones de los instrumentos separados generando los "armónicos", sonidos que no están presentes en ningún instrumento particular sino en su interacción.

Las resonancias de Poincaré conducen a una forma de caos, es decir, causas pequeñas tienen efectos grandes. En efecto, las simulaciones numéricas muestran que estas resonancias inducen la aparición de trayectorias erráticas, como sucede en el caos determinista, es decir, existe una estrecha analogía entre no integrabilidad y caos determinista. Ahora bien, la no integrabilidad tiene que ver con el azar, presente en el trabajo de Poincaré, cosa que Ilya Prigogine no comparte, ya que para él "las ecuaciones de sistemas caóticos son tan deterministas como las 
leyes de Newton. ¡Y empero engendran comportamientos de aspecto aleatorio!" (1996). Por el contrario, existen sistemas que no parecen caóticos no obstante estar regidos por el caos determinista. Es sobre esos sistemas que Mitchel Resnick ha investigado.

Resnick ha desarrollado su trabajo en torno a la teoría de sistemas de un modo práctico. Un ejemplo claro es la observación de los "tacos" de tránsito donde, si bien los autos avanzan el taco retrocede, ¿cómo es posible? El taco es más que un montón de autos, es un organismo diferente a la suma de los autos y tiene leyes propias e independientes, es decir existen diferentes "niveles" de organización.

En una ola lo que se mueve es la energía, no las moléculas del agua. De igual manera sucede con una onda en una cuerda: las partes de la cuerda se mueven hacia arriba y hacia abajo; la onda en cambio se traslada longitudinalmente. Los tacos vehiculares se pueden entender como otro tipo de olas: la densidad de los autos es análoga a la altura de la ola marina. En todos estos ejemplos, el movimiento del conjunto es bastante diferente de las partes que lo constituyen.

Frecuentemente se piensa en los niveles en términos de jerarquía: un general está a mayor nivel que un sargento y éste que un coronel. Pero también existen niveles de contenedor: un año contiene meses, y éstos días. Junto a estas dos definiciones tradicionales está la visión "emergente", que abarca los niveles que surgen de interacciones. Así, el taco de vehículos no está estructurado jerárquicamente, de hecho, su composición cambia constantemente, tampoco es una acumulación de autos, un año puede ser un mes largo, un taco es más que un auto grande.

El problema de la emergencia es que su aparición tiene que ver con el grado de apertura del sistema y, en esa medida, muchas veces es indeterminable, no obstante estar regido por las leyes de la complejidad.

Para muchos el análisis de la complejidad no es sólo una nueva ciencia, sino una forma de pensar la ciencia, un cambio de paradigma. Prigogine (1996) define esta nueva complejidad como la física del no equilibrio y sus postulados principales son:

1. Los procesos irreversibles (asociados a la flecha de tiempo) son tan reales como los procesos reversibles descritos por las leyes tradicionales de la física.
2. Los procesos irreversibles desempeñan un papel constructivo en la naturaleza.

3. La reversibilidad exige una extensión de la dinámica.

Así, la irreversibilidad crea nuevas formas de coherencia, la vida existe lejos del equilibrio.

\section{Sistemas Dinámicos como teoría Cognitiva}

Entre los años 80 y principios de los 90, sólo existían dos grandes posturas respecto a la cognición; una era la simbólica computacional basada en la linealidad y reversibilidad de los procesos. La otra, más reciente, era la conexionista (real, sobre procesadores paralelos, que diferiría de las primeras conexionistas de la era del Perceptron, denunciada ya por Minsky), en la que el procesamiento es paralelo y el símbolo es reemplazado por la representación.

Según algunos (Guinti, 1991), tanto las redes conexionistas como los algoritmos simbólicos son dinámicos en el sentido de ser sistemas que cambian a través del tiempo, Sin embargo, el dinamismo postula la irreversibilidad de los fenómenos a diferencia de aquellas. Así, si bien las redes neurales recursivas pueden ser dinámicas, la definición de conceptos como 'representación“ o 'computación' no está presente en la teoría de sistemas dinámicos.

La hipótesis dinamicista como la definen Van Gelder y Port, emplea sistemas de ecuaciones diferenciales para representar la trayectoria de los agentes cognitivos a través de los diferentes estados. En otras palabras, la cognición es explicada como un espacio multidimensional de todos los posibles pensamientos y conductas, que es atravesado por la ruta de las decisiones que toma un agente, bajo ciertas presiones ambientales e internas, todo lo cual es capturado por una serie de ecuaciones diferenciales (Van Gelder y Port 1995, citado por Eliasmith,1996).

Esta serie de ecuaciones está más cerca de los procesos irreversibles de Prigogine, aunque su base sea de tipo no lineal, es por esto que la postura ante definiciones de estados fijos como en la teoría clásica o la conexionista, es rechazada de plano por recurrir a un concepto emergente de la disposición e interacción de los constituyentes, que están definidos en su funcionamiento básico pero no en su interconexión. Thelen y Smith se refieren al problema de la 
representación en estos términos: "Nosotros afirmamos que la evolución sucede a causa de patrones de actividad fijados a través de componentes heterogéneos. No construimos representaciones del mundo conectando ideas temporalmente contingentes. ¡No construimos representaciones definitivamente! La mente es actividad en el tiempo... el tiempo real de las causas realmente físicas" (Thelen y Smith 1994, citado por Clark, 2001). Esto es claramente opuesto a los patrones conexionistas, con los que el dinamismo es comparado, en el sentido de que la definición de los patrones está dado no por la contingencia sino por la adaptabilidad, lo que sin duda genera un problema de lenguaje, ya que es el tiempo y no el espacio lo que define la actividad en los sistemas dinámicos.

Así, la evolución del pensamiento, que en el modelo simbólico computacional depende de un reloj externo para ejecutar su proceso serial (cristal de cuarzo), que en el modelo conexionista está presente por la disponibilidad de datos e instrucciones en los procesadores paralelos, en el modelo dinámico está regido sólo por el tiempo propio de respuesta al ambiente, y no por la existencia de instrucciones puestas allí por un programador. Por esto tampoco tienen un verdadero inicio: "son el tiempo".

Una de las mayores críticas hacia la postura dinamicista de la cognición es respecto a la construcción de modelos, si bien en otras áreas los modelos dinámicos pueden ser eficientes, al momento de modelar la cognición caen en la ambigüedad de denominar modelos claramente conexionistas (modelo de bulbo olfatorio) como ejemplos de sistemas dinámicos alejados del conexionismo y simbolismo. Claramente las redes conexionistas recursivas (una misma instrucción se usa más de una vez) son un modelo dinámico y también es cierto que las nociones de computación y representación se pueden aplicar al dinamismo.

Ahora bien, como los dinamicistas a) identifican solo cierto tipo de sistema capaz de describir la cognición (específicamente los sistemas de estados-determinados, donde la conducta es gobernada por ecuaciones diferenciales), y b) existen ecuaciones que (como lo demostró von Neumann) al ser reducidas se vuelven irreversibles, entonces la característica final de los sistemas que se aplican a la cognición tiene que ver con la irreversibilidad más que con los elementos constituyentes (redes neuronales) o la definición de representación. Esto es, hay aquí una postura más metafísica que procedural, que basa su experiencia en la imposibilidad de representar la inteligencia humana, aunque bien puede representar procesos cognitivos más simples como el comportamiento de una colonia de hormigas o el bulbo olfatorio de un conejo, es decir aquellos procesos que no impliquen autoconciencia o donde la innterrelación entre componentes sea tan extensa que se vuelva imposible de calcular. Entonces aparece una nueva duda: ¿Es posible representar la mente haciendo un modelo o solo se forma en la evolución de un cuerpo capaz de percibir? Si es lo último, se debería aceptar que la mente es fruto del desarrollo de variables tan complejas y multidimensionales que sólo se podría emular copiando un cuerpo exactamente, lo cual, por cierto, ya no sería un modelo.

Es lo que Kuhn señala al hablar de los "métodos ya dados", la existencia de "un grado de inconmensurabilidad entre los distintos modelos (relativamente excluyentes) y de lo problemático que resulta llevar el principio de la acumulación más allá de un determinado campo" (Kuhn 1994, citado por Monjeau, 2004). Es así que al forzar la aparición de un modelo que tenga relación con estructuras ya dadas y ser cuestionada por tener similitudes con esas estructuras, se verifica que: "La ciencia normal conduce sólo, en último análisis, al reconocimiento de anomalías y crisis. Y estas terminan, se resuelven, no mediante deliberación o interpretación, sino por un proceso repentino y no estructurado, como cambio de forma."

La dificultad de considerar como un nuevo paradigma a la teoría de sistemas dinámicos radica en que, como sistema, proponga solo un conjunto nuevo de metáforas o una nueva terminología para describir el fenómeno que otros ya han estado estudiando, y anunciar que este ha sido explicado cuando, en realidad, se ha sustituido el modelo por una analogía que, finalmente, tampoco explica nada sino que copia unas características del referente original.

De este modo el modelo, considerado una "clase de metáfora controlada" (Beardsley 1972, citado por Eliasmith 1996), no estaría presente en el trabajo de los dinamistas y sólo nos encontraríamos ante una analogía que mantiene una apertura hacia la interpretación y no define de manera absoluta sus propiedades. Barton, anuncia así que la descripción de conducta que 
hacen los dinamistas no es otra cosa que el análisis jungiano del comportamiento, maquillado con una formulación matemática. Sin embargo, esto podría decirse también de la postura simbólica computacional, que sí define una metodología basada en símbolos.

La explicación de toda conducta multidimensional es entendida, en un sistema dinámico, como una interacción de unas conductas de baja dimensionalidad de los agentes (equivalentes a los procesos irreversibles creativos). Así, programando un modelo con pocos tipos de conducta (avanzar, oler, girar), se pueden construir estructuras altamente dimensionales (colonias de hongos, tacos de tránsito). Sin embargo, en el modelado de la cognición las dimensiones de variables son prácticamente imposibles de determinar (por ser emergentes a su vez), lo que imposibilita la reducción (todavía) a una baja dimensionalidad de los sistemas de ecuación.

Es así como resulta muy difícil, aun con la tecnología actual, elaborar un modelo de pensamiento dinamicista en el que todas las variables de tipo biológico emulen al sistema nervioso. Un ejemplo más claro viene dado por la literatura, como veremos a continuación.

\section{Futuro posthumano y ciencia ficción}

Sin duda la física (y la filosofia) para tener algún valor debe ofrecer la posibilidad de sacar al hombre de su condición, de la tragedia de su existencia. Es así como la literatura recoge lo más liberador del dinamismo para ofrecer una alternativa a la tragedia de la existencia humana.

Sin embargo, aunque la teoría de los sistemas dinámicos resulta bastante poco habitual para el hombre común, y sea puesta en duda por los fundamentalistas, en la literatura de ciencia ficción es donde se ha aventurado más acerca de las posibilidades futuras de la humanidad y el conocimiento.

En la novela Schismatrix, Bruce Sterling imagina un sistema solar posthumano en el que los ingenieros genéticos han tomado el control de sus propios genes. En su etapa final la cultura alcanza el "quinto nivel de complejidad prigogínico", aludiendo a la teorización de Ilya Prigogine que señala que el orden emerge espontáneamente de los sistemas dinámicos cuando éstos se alejan mucho del equilibrio, dando lugar a puntos de transición llamados "singula- ridades". La teoría de Prigogine se aplica así a la humanidad, que es considerada como un sistema dinámico (los atractores pueden verse aquí como una clase de singularidad). Desplazándose lejos del equilibrio para convertirse en una clase post humana, la especie llega a una singularidad mística, una transcendencia final esquemáticamente descrita en este quinto nivel que está "tan lejos de la vida como la vida lo está de la materia" (Sterling 1985, citado por Dery, 1998).

En la misma línea Hans Moravec, director del Laboratorio de Robots Móviles del centro de Robótica de Carnegie-Mellon, cree con Marvin Minsky que la mente es una máquina de carne y que en esa línea es posible "descargar" la mente en un nuevo sistema interconectado, ya que la diferencia entre hombre y máquina es solo una cuestión de velocidad de cálculo. Esta velocidad se espera que alcance a los diez billones de operaciones por segundo en los próximos 10 años, y ya que la estructura cerebral determina el funcionamiento de la mente en todos los niveles, cada neurona y cada sinapsis en un cerebro dado deben recrearse en un programa de computador para que una conciencia individual pueda ser transmitida desde su cuerpo orgánico hacia una memoria digital $y$, puesto que en la teoría de la mente de Ross la conexión es el programa, no necesitamos comprender la conciencia humana para realizar esta operación.

Sin embargo, el físico Eric Harth sostiene que "la neurobiología y conciencia están inextricablemente unidas", lo que echaría por tierra la hipótesis de la "descarga del cerebro" planteada por Ross. Por supuesto, Harth arguye que, aunque admite que el cerebro no es un computador de uso general tipo Von Neumann, podría contener una conciencia humana si la emulación "llegase a un nivel lo suficientemente bajo (probablemente hasta neuronas individuales) para que emulase sistemas que están bajo el nivel esencial del cerebro" (Hart, 1996). Esto claramente es conexionismo.

Richard Restak, neurólogo, despeja esta discusión al preguntar: “ $¿ E s$ posible que nuestra definición de cerebro sea demasiado corta? ¿Acaso no están los procesos reguladores que situamos dentro de nuestras cabezas mucho más distribuidos?" (Restak 1991, citado por Dery, 1998). De este modo nos recuerda que los neurotransmisores y las hormonas reguladoras no se hallan confinados en el cerebro sino reparti- 


\section{MARCELO ARCE}

dos por todo el cuerpo, en los intestinos, en los pulmones, e incluso en los órganos sexuales. Y aunque pudiéramos extraer todas las conexiones, copiándolas con nanomáquinas, la conciencia no estaría tampoco ahí. Finalmente diremos con Félix Schwartzmann que "el curso evolutivo de las imágenes del mundo obedece a cierta compleja consistencia cognitiva que no cabe alterar." (Schwartzmann, 2000)

\section{Conclusión}

De este modo, podemos decir en defensa de la teoría de sistemas dinámicos que el que aún sus modelos no puedan ser construidos es debido al desarrollo de la tecnología y que para que el verdadero modelo de conciencia se materialice, es necesario incluir en los actuales las variables corporales (caóticas) que se han señalado. Tal vez entonces aparezcan las verdaderas falencias o la imposibilidad de reproducir el cerebro humano (en su concepción extendida de Restak) sin recurrir a sustitutos orgánicos. Pero lo que sí es cierto es que las denuncias del modelo dinámico son equivalentes a las que tuvo en sus orígenes la mecánica cuántica o la teoría del todo, guardando las debidas proporciones, en la que la verificación debió esperar años de desarrollo o reemplazar un sistema de medición por otro. Tal vez, como postula Beer (2000), sea necesaria una unión de las tres teorías actuales para generar una teoría válida, en lugar de buscar las diferencias en torno a procedimientos y definiciones, que es lo que actualmente se viene haciendo.

\section{Referencias}

BEER, RANDALL (2000). Dinamic aproaches to cognititive science. Trends in Cognitive Sciences, Vol.4, $\mathrm{N}^{\circ}$ 3, p.p. 91-98

CLARK, ANDY. (2001). Mindware, Oxford University Press, New York

DERY, MARK. (1998). Velocidad de Escape, Ediciones Siruela, Madrid.

ELIASMITH, C. (2001). Attractive and in-discrete: A critique of two putative virtues of the dynamicist theory of mind. Minds and Machines. $N^{\circ} 11$, p.p. 417-426

ELIASMITH, C. (1996). The third contender: A critical examination of the dynamicist theory of cognition. Philosophical Psycology. Vol. $9 \mathrm{~N}^{\circ}$ 4, p.p. 441-463

HAWKING, STEPHEN; PENROSE, ROGER. (1996). Naturaleza del espacio y el tiempo, Editorial Universitaria, Santiago.

JAQUES, ELLIOTT. (1984). La Forma del Tiempo, Paidos Studio, Buenos Aires.

MONJEAU, FEDERICO. (2004). La Invención Musical, Editorial Paidos, Buenos Aires.

PIAGET, J.; BRESSON, F.; RAISSE, P. Y OTROS. (1995). Introducción a la Psicolingüística, Ediciones Nueva Visión, Buenos Aires.

PRIGOGINE, ILYA. (1997). El fin de las certidumbres, Editorial Andrés Bello, Santiago.

SCHWARTZMANN, FÉLIX. (2000). Historia del universo y la conciencia, LOM Ediciones, Santiago. 